\title{
Intervention de santé publique face aux éclosions de grippe aviaire A(H5N2) et (H5N1) chez la volaille - Colombie-Britannique, décembre 2014 à février 2015 Murti $\mathbf{M}^{1^{\star}}$, Skowronski $D^{2}$, Lem $\mathbf{M}^{1}$, Fung $C^{1}$, Klar $\mathbf{S}^{1}$, Bigham $\mathbf{M}^{1}$, Loadman $\mathbf{S}^{1}$, Chambers $\mathrm{C}^{2}$, Pritchard $\mathrm{J}^{3}$,
}

${ }^{1}$ Fraser Health Authority, Surrey (Colombie-Britannique)

${ }^{2}$ Centre de contrôle des maladies de la Colombie-Britannique, Vancouver (Colombie-Britannique)

${ }^{3}$ Ministère de l'Agriculture, Abbotsford (Colombie-Britannique)

${ }^{*}$ Correspondance : Michelle.Murti@fraserhealth.ca

\section{Résumé}

En décembre 2014, la première détection au Canada d'un virus $A$ de la grippe aviaire hautement pathogène (IAHP) a été signalée chez la volaille dans la région de la Fraser Health Authority, en Colombie-Britannique. II s'agissait de la deuxième éclosion de grippe aviaire hautement pathogène (IAHP) provenant de virus réassortis H5 eurasiens en Amérique du Nord. La Fraser Health Authority a fourni la coordination primordiale de la santé publique pour cette intervention ainsi qu'une consultation et un soutien à l'égard de l'intervention en santé au travail.

L'intervention de santé publique était axée sur la recherche de sujets-contacts, la surveillance et le suivi des contacts familiaux, des contacts des ouvriers agricoles et d'autres contacts communautaires exposés dans les fermes touchées. Au total, on a identifié 50 sujets-contacts. Les sujets-contacts ont reçu une surveillance quotidienne active par des infirmières en santé publique pendant sept jours après leur dernière exposition, et on leur a conseillé de surveiller leurs symptômes jusqu'au jour 10. On a recommandé à tous les sujets-contacts et autres membres du ménage la vaccination contre la grippe saisonnière afin de se protéger contre un autre réassortiment possible avec les virus de la grippe humaine circulant au sein de la collectivité à ce moment-là. Au total, on a recommandé la chimioprophylaxie à 26 sujets-contacts (52\%) pour leur exposition continue aux granges et poulaillers touchés, et parmi eux, seules 11 personnes $(42 \%)$ ont entamé ce processus. Au cours de la période de surveillance active de sept jours, quatre sujets-contacts ont développé des symptômes respiratoires aigus et la grippe $B$ a été diagnostiquée chez une personne.

À l'échelle locale, les fournisseurs de soins de santé et les établissements de soins de courte durée ont été alertés de l'éclosion, et ils ont communiqué des messages publics au sujet des risques pour la santé humaine découlant du virus de la grippe aviaire. La collaboration entre les intervenants de la santé et de l'agriculture à l'échelle locale, régionale, provinciale et fédérale était essentielle pour une intervention rapide face à cette éclosion.

\section{Introduction}

En décembre 2014, la première détection en Amérique du Nord d'un virus de la grippe aviaire A hautement pathogène (IAHP) avec des gènes H5 de lignée eurasienne a été signalée chez la volaille dans la Fraser Health Authority, en Colombie-Britannique (Canada). II s'agissait d'un sous-type de virus de la grippe aviaire hautement pathogène $\mathrm{H} 5 \mathrm{~N} 2$ issu du mélange (c.-à-d. du réassortiment génétique) entre le virus de la grippe aviaire hautement pathogène H5N8 de la lignée eurasienne et le virus de la grippe aviaire faiblement pathogène de la lignée d'oiseaux sauvages d'Amérique du Nord.

La détection initiale a été suivie d'autres rapports sur des infections d'oiseaux domestiques et sauvages ou captifs par les virus réassortis de la grippe aviaire hautement pathogènes H5N8, H5N2 et H5N1 de la lignée eurasienne dans le Pacific Northwest des États-Unis en décembre 2014 et janvier 2015, ainsi que d'une détection 
supplémentaire du même nouveau virus réassorti de la grippe aviaire hautement pathogène H5N1 chez la volaille dans la FHA en février $2015(1,2)$.

Ces détections représentent la première éclosion de grippe aviaire hautement pathogène provenant de virus réassortis $\mathrm{H} 5$ eurasiens en Amérique du Nord. Le terme " hautement pathogène » désigne le spectre de la maladie observée chez les oiseaux, pas chez les humains. Les troupeaux touchés ont été touchés par une maladie grave et des taux de mortalité élevés, mais aucun cas d'infection humaine n'a été associé à ces virus H5. En général, les infections humaines par des virus de la grippe aviaire sont rares et ne se propagent pas facilement d'une personne à l'autre; cependant, la surveillance de la transmission entre les animaux et les hommes est essentielle pour surveiller le risque de maladie humaine, en particulier avec un nouveau virus réassorti. Le présent rapport fournit des détails liés à l'intervention en santé publique pour les détections des virus réassortis de la grippe aviaire hautement pathogènes H5N2 et H5N1 chez la volaille par la Fraser Health Authority.

\section{Infection par le virus de la grippe aviaire chez les oiseaux}

Entre le $1^{\text {er }}$ et le 19 décembre 2014, la Fraser Health Authority, située dans le sud-ouest de la région des bassesterres continentales de la Colombie-Britannique, qui est limitrophe des États-Unis, a été informée de l'infection de 11 élevages de volailles commerciaux et non commerciaux par le virus de la grippe aviaire hautement pathogène H5N2. Le rapport était le premier concernant la lignée eurasienne du virus de la grippe aviaire H5 hautement pathogène en Amérique du Nord. II s'agit de la deuxième éclosion d'une souche H5N2 d'IAHP en Amérique du Nord, après une éclosion en Pennsylvanie en 1983, et des éclosions antérieures causées par des virus d'IAHP H5N2 de la lignée nord-américaine avaient été signalées en Virginie-Occidentale en 2007, en ColombieBritannique en 2009, et au Manitoba en 2010 (3). L'analyse phylogénétique des virus H5N2 de I'IAHP associés à l'éclosion de 2014 révèle qu'il s'agit d'un nouveau virus réassorti A/turkey/BC/FAV10/2014 (H5N2) contenant des segments de gènes (numéros d'enregistrement GenBank : KP307954-KP307961) issus d'un virus d'IAHP H5N8 de la lignée eurasienne (y compris le gène $\mathrm{H} 5$ eurasien de variante génétique 2.3.4.4) et les segments ( $\mathrm{y}$ compris le gène N2) issus d'un virus d'IAHP typique de la lignée nord-américaine provenant des oiseaux sauvages $(4,5)$.

À la suite de l'éclosion du virus d'IAHP H5N2 en Colombie-Britannique, le ministère de l'Agriculture des ÉtatsUnis (USDA) a rapporté plusieurs détections de nouveaux virus réassortis d'IAHP de la lignée H5 eurasienne (H5N2, H5N8 et H5N1) chez les oiseaux domestiques et sauvages ou captifs dans les États de l'Oregon, de Washington, de l'Idaho, de l'Utah et de la Californie entre décembre 2014 et février 2015 (6). II s'agissait des premières détections des sous-types d'IAHP H5N8 et H5N1 chez des oiseaux en Amérique du Nord, et on pense qu'ils ont été introduits par le mélange d'oiseaux sauvages. Le 6 février 2015, la Fraser Health Authority a également été informée au sujet d'une ferme d'élevage non commercial de poules pondeuses où on avait détecté le virus d'IAHP H5N1 [A/Chicken/BC/FAV2/2015 (H5N1)]; le séquençage identifiait la même lignée d'oiseaux sauvages d'Amérique du Nord et un virus H5N1 presque identique, comme on l'avait détecté récemment dans l'État de Washington (1). Cette souche du virus H5N1 est génétiquement différent des virus de la grippe aviaire H5N1 qui avaient causé la maladie humaine dans d'autres pays.

Au total, les 11 fermes et deux élevages de basse-cour touchés au sein de la région comprenaient un mélange de dindes $(n=3)$, de poulets à griller $(n=7)$, de poules pondeuses (œufs de consommation) $(n=1)$ et de troupeaux non commerciaux ( $n=2$, y compris un avec un virus réassorti H5N1). Plus de 245000 oiseaux soit sont morts des suites de la maladie, soit ont été abattus sans cruauté dans le cadre de l'intervention de biosécurité contre les détections des virus d'IAHP H5N2 et H5N1 chez la volaille dans la région, coordonnée par l'Agence canadienne d'inspection des aliments (ACIA), le ministère de l'Agriculture de la Colombie-Britannique, ainsi que des organismes partenaires (7).

\section{Mesures d'intervention de santé publique}

En s'appuyant sur les éclosions de grippe aviaire précédentes dans la région, notamment l'expérience lors de l'éclosion du virus H7N3 en 2004, l'intervention de santé publique régionale de la FHA était axée sur la recherche des sujets-contacts, la surveillance et le suivi des contacts familiaux, des contacts des ouvriers agricoles et d'autres contacts communautaires exposés dans les fermes touchées $(8,9)$. La Fraser Health Authority n'a pas mené de recherches des sujets-contacts pour les entrepreneurs de l'Agence canadienne d'inspection des 
aliments $(\mathrm{ACI} A)$ et d'autres personnes exposées dans leur milieu de travail dans le cadre de l'intervention de biosécurité.

Au total, on a identifié 50 sujets-contacts dans les 13 établissements touchés : 35 (70\%) étaient des hommes, et l'âge médian était de 42 ans (fourchette de 12 à 75 ans). Les sujets-contacts ont été évalués selon leur exposition continue et la date de la dernière exposition à des oiseaux malades, et on les a informés sur les signes et les symptômes du syndrome grippal (SG) ainsi que sur la marche à suivre pour l'autosurveillance et la déclaration immédiate si des symptômes du SG se manifestaient. Les sujets-contacts fait l'objet d'une surveillance quotidienne active par des infirmières en santé publique pendant sept jours après leur dernière exposition, et on leur a conseillé de s'autosurveiller jusqu'au jour 10. Étant donné les mesures continues de destruction et d'élimination sur les lieux touchés, ils ont également reçu un appel téléphonique de suivi au jour 17 en vue de confirmer l'absence de nouvelles expositions et de s'assurer qu'ils restaient asymptomatiques.

Tous les sujets contacts et les autres membres du ménage se sont vus recommander la vaccination contre la grippe saisonnière afin de se protéger contre un autre réassortiment possible avec les virus de la grippe humaine circulant au sein de la collectivité à ce moment-là, notamment la grippe $A(H 3 N 2)(10)$. Au total, 26 personnes (52\%) se sont fait vacciner, soit elles l'avaient fait plus tôt dans la saison, soit elles l'ont fait au moment du suivi par les autorités de santé publique. On a recommandé aux sujets-contacts exposés de façon continue aux granges et poulaillers touchés au moment du premier suivi d'entamer une chimioprophylaxie antivirale à l'oseltamivir et de poursuivre avec les doses quotidiennes jusqu'à sept jours à partir de la dernière exposition. Au total, on a recommandé la chimioprophylaxie à 26 sujets-contacts (52\%), et parmi eux, seules 11 personnes (42\%) ont entamé la prophylaxie antivirale.

Au cours de la période de surveillance active de sept jours, quatre sujets-contacts ont développé des symptômes respiratoires - toux et nez qui coulait -, mais ils n'ont pas eu de fièvre, donc ils ne répondaient pas à la définition de cas de syndrome grippal. On a obtenu un écouvillonnage du nasopharynx pour les quatre sujets-contacts symptomatiques. Le virus de la grippe $B$ a été identifié chez une personne et les trois autres échantillons étaient négatifs pour le virus de la grippe $A$ et $B$. Deux des contacts symptomatiques ont entamé des doses de traitement d'oseltamivir avec l'apparition des symptômes par mesure de précaution, pendant que l'on attendait les résultats de l'écouvillonnage du nasopharynx.

À l'échelle locale, les fournisseurs de soins de santé et les établissements de soins de courte durée ont été alertés de l'éclosion début décembre, et ils ont fourni des recommandations au sujet de l'évaluation et de la prise en charge des patients asymptomatiques en lien avec des fermes d'élevage infectées. La Fraser Health Authority a également communiqué des messages publics sur les risques pour la santé humaine causés par le virus de la grippe aviaire dans les élevages de volailles et de porcs par l'entremise d'une coordination des activités avec les associations industrielles. Les messages comprenaient des recommandations sur l'immunisation contre la grippe saisonnière (financée par les fonds publics pour tous les ouvriers d'élevages de volaille en Colombie-Britannique) et l'usage systématique d'un équipement de protection individuelle. L'Agence canadienne d'inspection des aliments (ACIA) est le principal organisme de coordination de la santé et la sécurité au travail pour les personnes embauchées dans le cadre de l'intervention de biosécurité.

La collaboration entre les intervenants de la santé et de l'agriculture à l'échelle locale, régionale, provinciale et fédérale a permis une intervention rapide contre cette éclosion, et la FHA a dirigé la coordination de la santé publique, y compris la consultation et le soutien à l'égard de l'intervention en santé au travail.

\section{Conclusion}

La menace du virus de la grippe aviaire pathogène d'Asie (p. ex. H5N1 et H7N9) ayant un potentiel pandémique pour les humains appelle à la vigilance dans la définition de l'épidémiologie changeante et des nouveaux réassortiments. L'éclosion actuelle du virus d'IAHP H5N8 chez la volaille en Europe est la première détection de cette souche en Europe; elle avait déjà été détectée chez des oiseaux sauvages en Asie (11). La détermination d'un virus d'IAHP H5 de la lignée européenne en Amérique du Nord constitue une préoccupation pour l'introduction potentielle de la souche du virus zoonotique pathogène de la grippe aviaire provenant de l'Asie en Amérique du Nord. Avec des détections des virus H5N2 et H5N1 en Colombie-Britannique et H5N2, H5N8, et 
H5N1 dans cinq États du nord-ouest des États-Unis, il existe une probabilité accrue d'exposition humaine et d'infection au Canada (1).

Fin février 2015, on n'a pas relevé de cas humain de grippe H5N2, H5N8 ou H5N1 dans le cadre de la surveillance active associée aux éclosions récentes en Amérique du Nord. Cependant, certains virus de la grippe aviaire $\mathrm{H} 5$ peuvent causer une infection chez les humains, comme le montre l'éclosion généralisée du virus $\mathrm{A}(\mathrm{H} 5 \mathrm{~N} 1)$ en Asie et dans certaines régions de l'Afrique (718 cas/413 décès de janvier 2003 au 23 janvier 2015) (12).

À une échelle mondiale, presque tous les cas d'infection humaine par des virus de la grippe aviaire $\mathrm{H} 5$ ont été signalés chez des personnes en contact étroit avec des oiseaux infectés ou morts. Une contamination interhumaine est rare et le risque de propagation à l'échelle communautaire est faible. Par conséquent, les mesures de prévention mondiales se concentrent sur les personnes en contact très étroit avec les oiseaux contaminés. Les Centers for Disease Control and Prevention (CDC) ont publié des directives mises à jour pour les expositions humaines à ces types de souches (13). La surveillance continue des oiseaux (élevages et oiseaux sauvages) pour la grippe aviaire à déclaration obligatoire se poursuit au sein de la région touchée, y compris un suivi de santé publique en temps opportun pour toutes les personnes potentiellement exposées aux oiseaux infectés.

\section{Remerciements}

Les coauteurs remercient le personnel en santé publique, qui a contribué à la prise en charge des sujetscontacts, ainsi que les partenaires locaux, provinciaux, nationaux et internationaux qui ont participé à la présente intervention, notamment le $D^{r}$ John Pasick de l'Agence canadienne d'inspection des aliments. Ils aimeraient également remercier les éleveurs et d'autres intervenants concernés par cette éclosion.

\section{Conflit d'intérêts}

Aucun

\section{Financement}

Aucun

\section{Références}

(1) Jhung MA, Nelson DI. Outbreaks of avian influenza A (H5N2), (H5N8), and (H5N1) among birds - United States, December 2014-January 2015. MMWR. 2015;64(04):111.

(2) Pan American Health Organization (PAHO), World Health Organization (WHO). Epidemiological Alert. Detection and outbreaks of avian influenza due to reassortant viruses, public health implications for the Americas. Washington: PAHO;

http://www.paho.org/hq/index.php?option=com_docman\&task=doc_view\&gid=29005+\&ltemid=999999\&lang=en.

(3) World Animal Health Information Database (WĀHID) Interface Home Page. Paris: World Organization for Anmal Health; December 30, 2013. http://www.oie.int/wahis 2/public/wahid.php/Wahidhome/Home.

(4) Pasick J, Berhane Y, Joseph T, Bowes V, Hisanaga T, Handel K, Alexandersen S. Reassortant highly pathogenic influenza. $\mathrm{A}(\mathrm{H} 5 \mathrm{~N} 2)$ virus containing Eurasian lineage $\mathrm{H} 5$ and four other gene segments from Eurasian H5N8 in Canada, 2014. GenBank sequences deposited by Canadian Food Inspection Agency. Accession Numbers KP307954-KP307961.

(5) Ip HS, Torchetti MK, Crespo R, Kohrs P, DeBruyn P, Mansfield KG, et al. Novel Eurasian highly pathogenic influenza A H5 viruses in wild birds, Washington, USA, 2014. Emerg Infect Dis. 2015 May. http://dx.doi.org/10.3201/eid2105.142020

(6) US Department of Agriculture. Update on avian influenza findings in the Pacific Flyway. Washington: USDA; http://www.aphis.usda.gov/wps/portal/?urile=wcm:path:/aphis_content_library/sa_our_focus/sa_animal_health/sa_ani mal_disease_information/sa_avian_health. 
(7) Canadian Food Inspection Agency. Avian influenza - British Columbia infected premises

http://www.inspection.gc.ca/animals/terrestrial-animals/diseases/reportable/ai/2014-2015-ai-investigation-inbc/infected-premises/eng/1418340527324/1418340584180.

Disponible en français: http://www.inspection.gc.ca/animaux/animaux-terrestres/maladies/declarationobligatoire/ia/2014-2015-enquete-de-ia-en-cb/lieux-infectes/fra/1418340527324/1418340584180.

(8) Skowronski DM, Li Y, Tweed A, Tam TWS, Petric M, David ST, Larder A, Birch P, Marra F, Seto J, Bastien N, King A, Lee SW, Prendergast $P$, Krajden M, Brunham R. Protective measures and human antibody response during an avian influenza H7N3 outbreak in poultry in British Columbia, Canada. CMAJ. 2007;176:47-53.

(9) Tweed SA, Skowronski DM, David ST, Larder A, Petric M, Lees M, et al. Human illness from avian influenza H7N3, British Columbia. Emerg Infect Dis. 2004;10:2196-9.

(10) BC Centre for Disease Control [Internet]. Influenza surveillance reports. Vancouver: BCDC; 2015. http://www.bccdc.ca/dis-cond/DiseaseStatsReports/influSurveillanceReports.htm.

(11) European Centre for Disease Prevention and Control (ECDC). Outbreaks of highly pathogenic avian influenza $\mathrm{A}(\mathrm{H} 5 \mathrm{~N} 8)$ in Europe. Solna Sweden: ECDC; November 20, 2014.

http://www.ecdc.europa.eu/en/publications/Publications/H5N8-influenza-Europe-rapid-risk-assessment-20-

November-2014.pdf.

(12) World Health Organization (WHO). Influenza at the human-animal interface. Summary and assessment as of 26 January 2015. Geneva: WHO; 2015.

http://www.who.int/influenza/human_animal_interface/Influenza_Summary_IRA_HA_interface_26January2015.pdf.pd f?ua=1.

(13) Centers for Disease Control and Prevention (CDC) Interim guidance on testing, specimen collection, and processing for patients with suspected infection with novel influenza A viruses with the potential to cause severe disease in humans. Atlanta: CDC;

http://www.cdc.gov/flu/avianflu/severe-potential.htm. 